Calculation of the stark widths and shifts for several lines of the thallium atom

This article has been downloaded from IOPscience. Please scroll down to see the full text article. 1996 Phys. Scr. 54332

(http://iopscience.iop.org/1402-4896/54/4/004)

View the table of contents for this issue, or go to the journal homepage for more

Download details:

IP Address: 138.100.132.20

The article was downloaded on 17/02/2012 at 09:16

Please note that terms and conditions apply. 


\title{
Calculation of the Stark Widths and Shifts for Several Lines of the Thallium Atom
}

\author{
A. Alonso-Medina ${ }^{1 *}$ and C. Herrán-Martínez ${ }^{2}$ \\ ${ }^{1}$ Dpto. de Física Atómica, Molecular y Nuclear. \\ 2 Dpto. de Física de la Tierra, Astronomía y Astrofisica II, Facultad de CC.Físicas. Universidad Complutense de Madrid. Avda Complutense s/n. 28040 \\ Madrid, Spain.
}

Received October 3, 1995; accepted February 13, 1996

\begin{abstract}
Stark widths and shifts for eight lines of $T I I$, arising from the $n \mathrm{p}^{2} \mathbf{P}_{1 / 2,3 / 2}^{0}$ $(n=7-9)$ and $7 \mathrm{~s}^{2} \mathrm{~S}_{1 / 2}$ levels have been calculated using semiempirical formulas, and compared with those obtained by other authors.
\end{abstract}

\section{Introduction}

Stark parameters of spectral lines are necessary for solving astrophysical problems that need to accomplish laboratory plasma diagnostics and to verify the broadening of spectral lines. Quite a large number of articles have been published that use different theoretical approximations to calculate these parameters and to study their dependence on the nuclear charge number, effective ionization potential, atomic polarizability or principal quantum number, [1-4], but very few papers have calculated and studied these parameters specifically for $\mathrm{Tl}$, and there is barely any data on these parameters.

In this paper we present Stark widths and shifts calculated for eight lines of T1 I using Griem's semiclassical calculations, [5], for different electron densities and plasma temperatures; for most of these lines there is no data in the bibliography. Moore's tables [6] have been used to obtain the level energies; Fig. 1 displays a partial scheme of the thallium atom energy levels.

The values obtained in this paper have been compared with the values of other authors, [7] and [8], and are in good agreement. Lakicevic, [7], obtained the Stark parameter values of the resonance line of $3775.7 \AA$ (air), $7 \mathrm{~s} \rightarrow 6 \mathrm{p}$, for an electron density of $10^{17} \mathrm{~cm}^{-3}$ and an electron temperature of $20 \times 10^{3} \mathrm{~K}$. The measurement made by Couris et al. $[8,9]$ of the Stark shift for the line at $6551.7 \AA$ obtained in a plasma with an electron density of $0.004 \times 10^{17} \mathrm{~cm}^{-3}$ and an electron temperature of $6000 \mathrm{~K}$, is the first Stark broadening experimental data measured for this line.

\section{Theory}

In 1968 Griem [5], suggested a simple semiempirical impact approximation based on Barangers original formulation [10], together with the use of an effective Gaunt-factor approximation proposed by Seaton [11], and Van Regemorter [12]. The Stark line width can be calculated from

*E-mail: Aurelia@eucmax.sim.ucm.es

Physica Scripta 54 the following semiempirical formula:

$$
\begin{aligned}
\omega \approx & 8\left(\frac{\pi}{3}\right)^{3 / 2} \frac{\hbar}{m a_{0}} N_{e}\left(\frac{E_{\mathrm{H}}}{k T}\right)^{1 / 2} \\
& \times \sum_{i^{\prime} f^{\prime}}\left[\left|\left\langle i^{\prime}|r| i\right\rangle\right|^{2} \bar{g}_{\omega}\left(x_{i^{\prime}}\right)+\left|\left\langle f^{\prime}|r| f\right\rangle\right|^{2} \bar{g}_{\omega}\left(x_{f^{\prime} f}\right)\right]
\end{aligned}
$$

and the shifts can be calculated from a formula similar to eq. (1):

$$
\begin{aligned}
d \approx & -8\left(\frac{\pi}{3}\right)^{3 / 2} \frac{\hbar}{m a_{0}} N_{\mathrm{e}}\left(\frac{E_{\mathrm{H}}}{k T}\right)^{1 / 2} \\
& \times \sum_{i^{\prime} f^{\prime}}\left[\frac{\Delta E_{i^{\prime} i}}{\left|\Delta E_{i^{\prime} i}\right|}\left|\left\langle i^{\prime}|r| i\right\rangle\right|^{2} \bar{g}_{\mathrm{sh}}\left(x_{i^{\prime} i}\right)\right. \\
& \left.-\frac{\Delta E_{f^{\prime} f}}{\left|\Delta E_{f^{\prime} f}\right|}\left|\left\langle f^{\prime}|r| f\right\rangle\right|^{2} \bar{g}_{\mathrm{sh}}\left(x_{f^{\prime} f}\right)\right] .
\end{aligned}
$$

The Stark parameters calculated by these formulas are given in units of frequency; $E_{\mathrm{H}}$ is the Hydrogen ionization energy, $N_{\mathrm{e}}$ is the electron density, $m$ is the electron mass, $k$ is the Boltzmann constant, $a_{0}$ is the Bohr radius, $T$ the electron temperature, $E=3 / 2 k T$ the energy of the perturbing electron, and $\bar{g}_{\omega}$ and $\bar{g}_{\text {sh }}$ are the effective Gaunt-factors for widths and shifts respectively, which are slowly varying

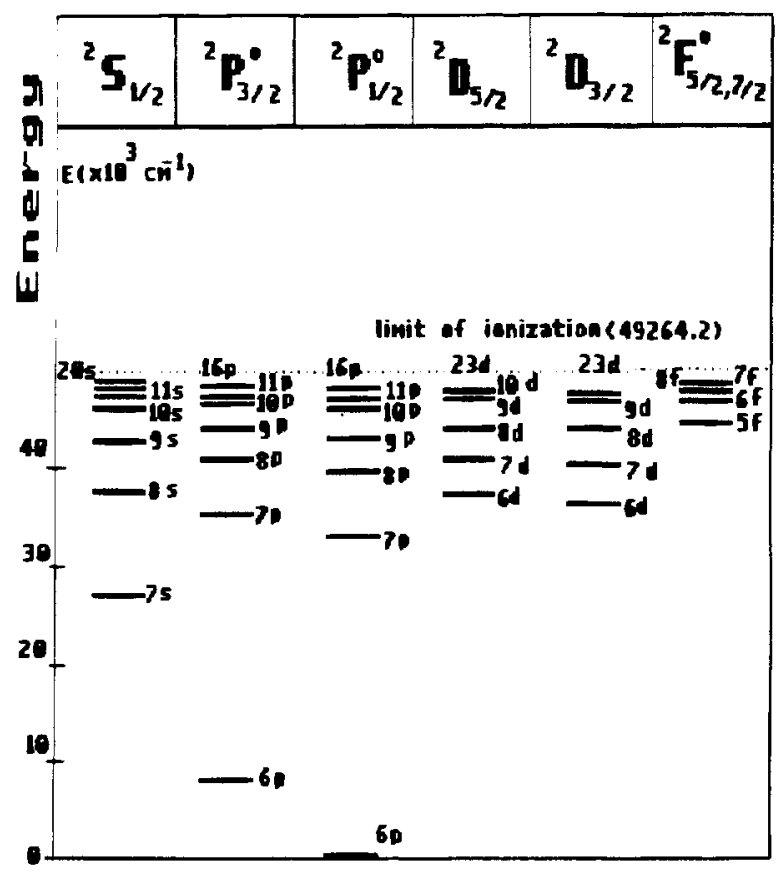

Fig. 1. Partial diagram of energy levels of the thallium atom. 
Table I. Parameters of Stark broadening of lines of Tallium $\left[N_{\mathrm{e}}=0.004 \times 10^{17} \mathrm{~cm}^{-3} \text { and } 1 \times 10^{17} \mathrm{~cm}^{-3}\right]^{1}$.

\begin{tabular}{|c|c|c|c|c|c|c|c|}
\hline \multirow{2}{*}{$\begin{array}{l}\lambda(\AA) \\
\text { vacuum }\end{array}$} & \multirow[b]{2}{*}{ Transition } & \multirow{2}{*}{$\begin{array}{l}\text { Temperature } \\
\left(\times 10^{3} \mathrm{~K}\right)\end{array}$} & \multicolumn{3}{|c|}{ Data of this paper } & \multicolumn{2}{|c|}{ Data of bibliography } \\
\hline & & & $\omega(\AA)$ & $d(\AA)$ & $d / \omega$ & $\omega(\AA)$ & $d(\AA)$ \\
\hline \multirow[t]{4}{*}{13016.8} & \multirow{4}{*}{$7 \mathrm{p}^{2} \mathrm{P}_{1 / 2}^{0} \rightarrow 7 \mathrm{~s}^{2} \mathrm{~S}_{1 / 2}$} & 6 & $0.0067-16.7$ & $0.0010-2.4$ & $0.15-0.14$ & & \\
\hline & & 10 & $0.0058-14.5$ & $0.0015-3.6$ & $0.26-0.25$ & & \\
\hline & & 15 & $0.0056-13.9$ & $0.0019-4.7$ & $0.34-0.34$ & & \\
\hline & & 20 & $0.0055-13.7$ & $0.0018-4.5$ & $0.33-0.33$ & & \\
\hline \multirow[t]{4}{*}{11516.0} & \multirow{4}{*}{$7 p^{2} P_{3 / 2}^{0} \rightarrow 7 s^{2} S_{1 / 2}$} & 6 & $0.0092-23.0$ & $0.0059-14.9$ & $0.64-0.65$ & & \\
\hline & & 10 & $0.0091-22.9$ & $0.0061-15.3$ & $0.67-0.67$ & & \\
\hline & & 15 & $0.0086-21.5$ & $0.0056-14.0$ & $0.65-0.65$ & & \\
\hline & & 20 & $0.0085-21.2$ & $0.0056-14.0$ & $0.66-0.66$ & & \\
\hline \multirow[t]{4}{*}{6715.6} & \multirow[t]{4}{*}{$8 \mathrm{p}^{2} \mathrm{P}_{1 / 2}^{0} \rightarrow 7 \mathrm{~s}^{2} \mathrm{~S}_{1 / 2}$} & 6 & $0.0084-21.0$ & $0.0059-14.6$ & $0.70-0.69$ & & \\
\hline & & 10 & $0.0087-21.8$ & $0.0060-15.0$ & $0.69-0.69$ & & \\
\hline & & 15 & $0.0084-21.1$ & $0.0051-12.7$ & $0.61-0.60$ & & \\
\hline & & 20 & $0.0082-19.3$ & $0.0045-10.7$ & $0.55-0.55$ & & \\
\hline \multirow[t]{4}{*}{6551.7} & \multirow{4}{*}{$8 p^{2} P_{3 / 2}^{0} \rightarrow 7 s^{2} S_{1 / 2}$} & 6 & $0.024-46.8$ & $0.021-40.0$ & $0.87-0.85$ & & $0.025-$ \\
\hline & & 10 & $0.018-45.0$ & $0.013-33.0$ & $0.72-0.73$ & & (Couris et al., 1988) \\
\hline & & 15 & $0.019-47.2$ & $0.011-26.7$ & $0.56-0.56$ & & \\
\hline & & 20 & $0.018-45.3$ & $0.010-25.4$ & $0.56-0.56$ & & \\
\hline \multirow[t]{4}{*}{5585.5} & \multirow{4}{*}{$9 p^{2} \mathrm{P}_{1 / 2}^{0} \rightarrow 7 \mathrm{~s}^{2} \mathrm{~S}_{1 / 2}$} & 6 & $0.023-58.2$ & $0.014-35.2$ & $0.61-0.60$ & & \\
\hline & & 10 & $0.023-57.8$ & $0.010-26.1$ & $0.44-0.45$ & & \\
\hline & & 15 & $0.023-58.8$ & $0.010-25.5$ & $0.43-0.43$ & & \\
\hline & & 20 & $0.022-54.6$ & $0.0086-21.4$ & $0.39-0.39$ & & \\
\hline \multirow[t]{4}{*}{5519.4} & \multirow[t]{4}{*}{$9 \mathrm{p}^{2} \mathrm{P}_{3 / 2}^{0} \rightarrow 7 \mathrm{~s}^{2} \mathrm{~S}_{1 / 2}$} & 6 & $0.050-124.9$ & $0.037-91.7$ & $0.74-0.73$ & & \\
\hline & & 10 & $0.045-112.3$ & $0.029-72.7$ & $0.64-0.65$ & & \\
\hline & & 15 & $0.042-104.9$ & $0.024-60.7$ & $0.57-0.58$ & & \\
\hline & & 20 & $0.041-103.5$ & $0.021-51.6$ & $0.51-0.50$ & & \\
\hline \multirow[t]{4}{*}{3775.7} & \multirow{4}{*}{$7 s^{2} S_{1 / 2} \rightarrow 6 p^{2} P_{1 / 2}^{0}$} & 6 & $2.1 \times 10^{-4}-0.53$ & $1.2 \times 10^{-4}-0.31$ & $0.57-0.58$ & & \\
\hline & & 10 & $1.6 \times 10^{-4}-0.44$ & $1.1 \times 10^{-4}-0.26$ & $0.60-0.59$ & & \\
\hline & & 15 & $1.3 \times 10^{-4}-0.33$ & $1.0 \times 10^{-4}-0.20$ & $0.59-0.60$ & & \\
\hline & & 20 & $1.2 \times 10^{-4}-0.30$ & $0.7 \times 10^{-4}-0.17$ & $0.58-0.57$ & $\begin{array}{l}-0.29 \\
\text { (Lekicevic, 1983) }\end{array}$ & $\begin{array}{l}-0.155 \\
\text { (Lekicevic, 1983) }\end{array}$ \\
\hline \multirow[t]{4}{*}{5350.5} & \multirow[t]{4}{*}{$7 \mathrm{~s}^{2} \mathrm{~S}_{1 / 2} \rightarrow 6 \mathrm{p}^{2} \mathrm{P}_{3 / 2}^{0}$} & 6 & $4.9 \times 10^{-4}-1.22$ & $2.2 \times 10^{-4}-0.55$ & $0.45-0.45$ & & \\
\hline & & 10 & $3.8 \times 10^{-4}-0.44$ & $1.9 \times 10^{-4}-0.47$ & $0.50-0.50$ & & \\
\hline & & 15 & $3.1 \times 10^{-4}-0.79$ & $1.8 \times 10^{-4}-0.47$ & $0.58-0.59$ & & \\
\hline & & 20 & $2.9 \times 10^{-4}-0.73$ & $1.7 \times 10^{-4}-0.43$ & $0.59-0.59$ & & \\
\hline
\end{tabular}

${ }^{1}$ In each cell with the data of this paper or of the bibliography are two values, as for example $0.0067-16.7$ in the first cell of fourth column, $\omega(\AA)$; it means that $\omega(\AA)=0.067$ when $N_{\mathrm{o}}=0.004 \times 10^{17} \mathrm{~cm}^{-3}$, and $\omega(\AA)=16.7$ when $N_{\mathrm{o}}=1 \times 10^{17} \mathrm{~cm}^{-3}$.

functions of $x_{i^{\prime} i}=E / \Delta E_{i^{\prime} i}$, where $\Delta E_{i^{\prime} i}$ is the energy difference between perturbing level $i^{\prime}$ and perturbed level $i$; the indices $i$ and $f$ denote the initial (upper) and final (lower) levels of the transition, respectively; these factors had been calculated by Griem, [5].

$\left\langle i^{\prime}|r| i\right\rangle$ is the atomic dipole matrix element connecting the initial $(i)$ and final $\left(i^{\prime}\right)$ atomic states in the inelastic colli-

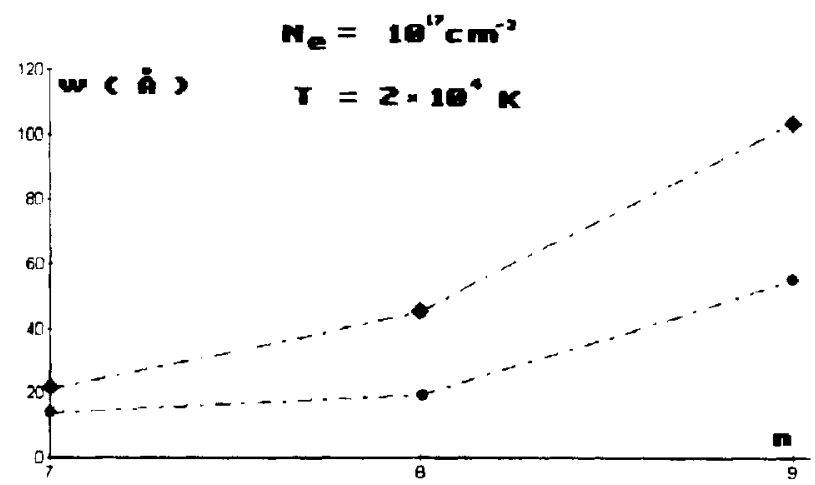

Fig. 2. Stark widths vs. principal quantum number in two series of $\mathrm{Tl} I$. $n \mathrm{p}^{2} \mathrm{P}_{3 / 2}^{0} \rightarrow 7 \mathrm{~s}^{2} \mathrm{~S}_{1 / 2} ;: n \mathrm{p}^{2} \mathrm{P}_{1 / 2} \rightarrow 7 \mathrm{~s}^{2} \mathrm{~S}_{1 / 2}$. sion. The matrix elements in eqs (1) and.(2) were calculated using a semiempirical relativistic one-electron central-field model potential; the equation for the dipole length

$\int_{0}^{\infty} P_{n l}(r) r P_{n^{\prime} l^{\prime}}(r) \mathrm{d} r$

was used to calculate the transition integrals.

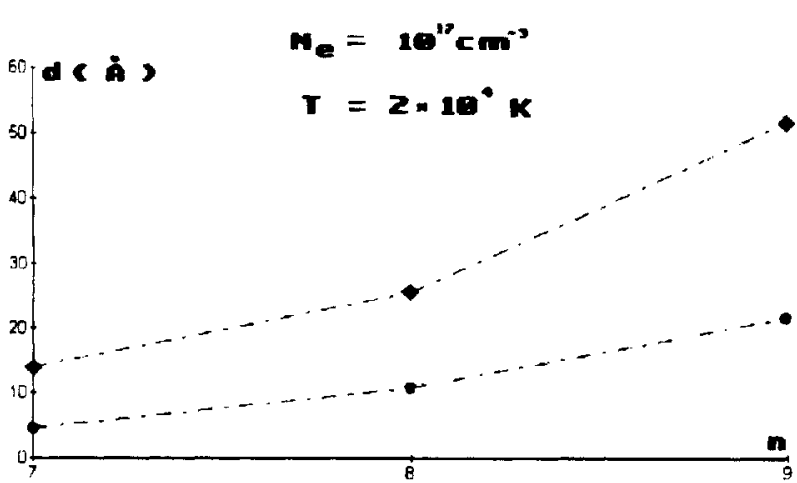

Fig. 3. Stark shifts $v$ s. principal quantum number in two series of $\mathrm{Tl}$ I. $n \mathrm{p}^{2} \mathrm{P}_{3 / 2}^{0} \rightarrow 7 \mathrm{~s}^{2} \mathrm{~S}_{1 / 2} ; \bullet: n \mathrm{p}^{2} \mathrm{P}_{1 / 2} \rightarrow 7 \mathrm{~s}^{2} \mathrm{~S}_{1 / 2}$. 
The radial wave functions $P_{n c}(r)$ were obtained by numerical integration of the radial Schrödinger equation including three terms in the Hamiltonian [13],

$-\frac{\alpha^{2}}{2}(E-V)^{2}-\frac{\alpha^{2}}{4}\left(\frac{\mathrm{d} V}{\mathrm{~d} r}\right) r \frac{\mathrm{d}}{\mathrm{d} r} r^{-1}+\frac{\alpha^{2}}{2}\left(\frac{\mathrm{d} V}{\mathrm{~d} r}\right) \frac{l s}{r}$,

where $\alpha$ is the fine structure constant, $E$ is the eigenvalue of the Schrödinger equation, $l$ and $s$ are orbital and spin angular-momentum operators and $V$ a semiempirical potential given by Green et al. [14], that includes core polarization effects [15], and include the finite size of the nucleus.

The core polarization potential,

$V_{p}(r)=-\frac{1}{2} \alpha_{p} \frac{r^{2}}{\left(r^{2}+r_{0}^{2}\right)^{3}}$,

is included in the one-electron Hamiltonian of each valence electron. The static dipole polarizability of TlI is set to the value ( 39.33 a.u.) calculated by Fraga et al. [16], and $r_{0}$ is equated to the mean $(2.38 \mathrm{a}$.u.) of the outermost $(n l j)$ orbital of the unpolarized parent ion. The matrix element is corrected for core polarization by replacing the dipole moment operator of the valence electron by

$D(r)=r\left[1-\alpha_{p}\left(r^{2}+r_{0}^{2}\right)^{-3 / 2}\right]$.

To incorporate the finite size of the nucleus, the potential term is given for

$V(r)=\frac{Z}{2 R_{0}^{3}} r^{2}-\frac{3}{2} \frac{Z}{R_{0}}$,

where $R_{0}$ is the radius of the nucleus [17].

\section{Results and discussion}

The values of the Stark parameters, width $\omega$ and shift $d$, calculated in this paper, are displayed in Table I, expressed in $\AA$ wavelength units to permit comparison with the values given by other authors. The parameters have been calculated in the electron temperature range $(6-20) \times 10^{3} \mathrm{~K}$ and electron densities of $0.004 \times 10^{17} \mathrm{~cm}^{-3}$ and $1 \times 10^{17} \mathrm{~cm}^{-3}$.

The semiempirical values of $\omega(\AA)$ are shown in the fourth column, the $d(\AA)$ values in the fifth column and the $d / \omega$ quotient values are in the sixth column. Each cell of these columns contains four pairs of values; each pair corresponds to a different temperature, and the values of each pair are the parameters calculated for the values of the electron density, $0.004 \times 10^{17} \mathrm{~cm}^{-3}$ and $1 \times 10^{17} \mathrm{~cm}^{-3}$ respectively. The table also includes the few values found in the bibliography for these parameters $[7,8]$, and as may be seen, extremely similar to those calculated in this paper; these values are shown in the table in accordance with the criteria applied to those obtained in this paper.

Equations (1) and (2), used in the calculations, explain different dependencies of the Stark parameter values calculated on the different variables used, such as for example that for given values of $N_{\mathrm{e}}$ and $T_{\mathrm{e}}$. The value of the parameters grow regularly with the principal quantum number in the spectral series $n p^{2} \mathrm{P}_{3 / 2}^{0} \rightarrow 7 \mathrm{~s}^{2} \mathrm{~S}_{1 / 2}$ and $n \mathrm{p}^{2} \mathrm{P}_{1 / 2} \rightarrow 7 \mathrm{~s}^{2} \mathrm{~S}_{1 / 2}$. This is to be expected since in this way the initial level is higher and therefore the energy difference between the perturbed levels is lower; these variations are shown in Figs 2 and 3.

This type of dependence has also been studied and verified in other spectral series of different atomic species, [1-4].

\section{Acknowledgements}

The authors wish to thank Prof. Dr. J. Campos, Director of the Department, and Prof. Dr. C. Colon for their numerous and valuable comments and suggestions.

\section{References}

1. Roberts, D. E. and Bernard, A. J., J. Quant. Spectrosc. Radiat. Transf. 12, 1205 (1972).

2. Puric, J., Lakićević, I. S. and Glavonic, V., J. Quant. Spectrosc. Radiat. Transf. 26, 65 (1981).

3. Puric, J., Cuk, M. and Lakićević, I. S., Phys. Rev. A32, 1106 (1985).

4. Wiese, W. L. and Konjevic, N., J. Quant. Spectrosc. Radiat. Transf. 47, 185 (1992).

5. Griem, H. R., Phys. Rev. 165, 258 (1968).

6. Moore, C. E., "Atomic Energy Levels" NBS 467, 11e U.S. GPO (Washington D.C. 1958).

7. Lakicevic, L. S., Astron. Astrophys. 127, 37 (1983).

8. Couris, S., Damelincourt, J. J., Drakakis, E. and Karabourniotis, D., "The Physics of Ionized Gases SPIG" (Electrical Engineering Faculty, Sarajevo 1988), p. 325.

9. Konjevic, N. and Wiese, W. L., J. Phys. Chem. Ref. Data 19, 1380 (1990).

10. Baranger, M., Phys. Rev. 112, 855 (1958).

11. Seaton, M. J., "Atomic and Molecular Processes" (Edited by D. R. Bates) (Academic Press, New York 1962), chap. 11.

12. Van Regemorter, H., Astrophys. J. 136, 906 (1962).

13. Cowan, R. D., "The Theory of Atomic Structure and Spectra" (University of California Press, London 1981).

14. Green, A. E. S., Sellin, D. L. and Zachor, A. S., Phys. Rev. 184, 1 (1969).

15. Migdalek, J. and Baylis, W. E., J. Phys. B: At. Mol. Phys. 12, 2595 (1979).

16. Fraga, S., Karwowski, J. and Saxena, K. M. S., "Handbook of Atomic Data" (Elsevier Scientific Publishing Company, New York 1976), p. 323 and 469.

17. Alonso-Medina, A., Physics Doctoral Thesis, Fac. de Ciencias Físicas. Universidad Complutense, Madrid, 1995. 